\title{
NASA Advanced Explorations Systems: Concepts for Logistics to Living
}

\author{
Sarah A. Shull \\ NASA, Johnson Space Center, Houston, TX \\ A. Scott Howe, PhD \\ NASA, Jet Propulsion Laboratory, Pasadena, CA \\ Michael T. Flynn, PhD \\ NASA, Ames Research Center, Moffett Field, CA \\ Robert Howard, PhD \\ NASA, Johnson Space Center, Houston, TX
}

The NASA Advanced Exploration Systems (AES) Logistics Reduction and Repurposing (LRR) project strives to enable a largely mission-independent cradle-tograve-to-cradle approach to minimize logistics contributions to total mission architecture mass. The goals are to engineer logistics materials, common crew consumables, and container configurations to meet the following five basic goals:

1. Minimize intrinsic logistics mass and improve ground logistics flexibility.

2. Allow logistics components to be directly repurposed for on-orbit non-logistics functions (e.g., crew cabin outfitting) - thereby indirectly reducing mass/volume.

3. Compact and process logistics that have not been directly repurposed to generate useful on-orbit components and/or compounds (e.g., radiation shielding, propellant, other usable chemical constituents).

4. Enable long-term stable storage and disposal of logistics end products that cannot be reused or repurposed (e.g., compaction for volume reduction, odor control, and maintenance of crew cabin hygienic conditions).

5. Allow vehicles in different mission phases to share logistics resources.

This paper addresses the work being done to meet the second goal, the direct repurposing of logistics components to meet other on-orbit needs, through a strategy termed Logistics to Living (L2L). L2L has several areas but can be defined as repurposing or converting logistical items (bags, containers, foam, components, etc.) into useful crew items or life support augmentation on-orbit after they have provided their primary logistics function. The intent is that by repurposing items, dedicated crew items do not have to be launched and overall launch mass is decreased. For non-LEO missions, the vehicle interior volume will be relatively fixed so L2L will enable this volume to be used more effectively through reuse and rearrangement of logistical components.

Past work in the area of L2L has already conceptually developed several potential technologies [Howe, Howard 2010]. Several of the L2L concepts that have shown the most potential in the past are based on NASA cargo transfer bags (CTBs) or their equivalents which are currently used to transfer cargo to and from the ISS. A high percentage of all logistics supplies are packaging mass and for a 6-month mission a crew of four might need over 100 CTBs. These CTBs are used for on-orbit transfer and storage 
but eventually becomes waste after use since down mass is very limited. The work being done in L2L also considering innovative interior habitat construction that integrate the CTBs into the walls of future habitats. The direct integration could provide multiple functions: launch packaging, stowage, radiation protection, water processing, life support augmentation, as well as structure. Reuse of these CTBs would reduce the amount of waste generated and also significantly reduce future up mass requirements for exploration missions.

Also discussed here is the L2L "water wall", an innovative reuse of an unfolded CTB as a passive water treatment system utilizing forward osmosis. The bags have been modified to have an inner membrane liner that allows them to purify wastewater. They may also provide a structural water-wall element that can be used to provide radiation protection and as a structural divider. Integration of the components into vehicle/habitat architecture and consideration of operations concepts and human factors will be discussed. In the future these bags could be designed to treat wastewater, concentrated brines, and solid wastes, and to dewater solid wastes and produce a bio-stabilized construction element.

This paper will describe the follow-on work done in design, fabrication and demonstrations of various L2L concepts, including advanced CTBs for reuse/repurposing, internal outfitting studies and the CTB-based forward osmosis water wall.

\section{References}

Howe, A. Scott, Howard, Robert Dual Use of Packaging on the Moon: Logistics-2Living, AIAA-2010-6049, 40th International Conference on Environmental Systems, 11 15 Jul 2010. Barcelona, Spain. 